\title{
Study of Longitudinal Oscillations of a Building on the Basis of Continuum Plate Model
}

\author{
Makhamatali K. Usarov, Gayratjon T. Ayubov \\ Institute of Mechanics and Seismic Stability of Structures, Academy of Sciences of Uzbekistan, Tashkent, Uzbeksitan \\ Email: umakhamatali@mail.ru
}

How to cite this paper: Usarov, M.K. and Ayubov, G.T. (2018) Study of Longitudinal Oscillations of a Building on the Basis of Continuum Plate Model. Journal of Applied Mathematics and Physics, 6, 1928-1936. https://doi.org/10.4236/jamp.2018.69164

Received: September 3, 2018

Accepted: September 27, 2018

Published: September 30, 2018

Copyright (c) 2018 by authors and Scientific Research Publishing Inc. This work is licensed under the Creative Commons Attribution International License (CC BY 4.0).

http://creativecommons.org/licenses/by/4.0/

(c) (i) Open Access

\begin{abstract}
Continuum plate model in the form of a cantilever anisotropic plate developed in the framework of the bimoment theory of plates describing seismic oscillations of buildings is proposed in this paper as a dynamic model of a building. Formulas for the reduced moduli of elasticity, shear and density of the plate model of a building are given. Longitudinal oscillations of a building are studied using the continuum plate and box-like models of the building with Finite Element Model. Numerical results are obtained in the form of graphs, followed by their analysis.
\end{abstract}

\section{Keywords}

Seismic Effects, Continuum Plate Model of a Building, Cantilever Plate, Moment, Bimoment, Boundary Conditions, Finite Element Method (FEM) and Finite Difference Method (FDM)

\section{Introduction}

Among numerous objects of study in mechanics of a deformed rigid body, multi-storey buildings and structures occupy a special place. Development of dynamic models of buildings and structures with consideration of strain of spatial nature is one of the most difficult and urgent problems in mechanics.

A universal model of the building has not been developed yet. This is due to the complex structure, diversity and numerous elements of the building, especially tall large-sized buildings. Therefore, one of the important tasks of modern mechanics and seismic stability of structures is the development of universal models of a building that adequately describes its spatial behavior.

There are numerous articles and monographs devoted to the development of the theory of seismic resistance of a building. Various methods for calculating buildings and structures for seismic actions have been developed, taking into 
account important factors, such as seismic loading, soil conditions of terrain and structural features of buildings [1]-[6]. Note that the analysis of the consequences of strong earthquakes has shown the shortcomings of existing methods of calculating buildings and structures for seismic resistance. One of the most common design schemes of the building is a multi-mass elastic cantilever rod. Oscillations of a spatial construction are reduced to the consideration of oscillations of a plane system consisting of several concentrated masses connected by certain rigidities. Many researchers, criticizing the cantilever design of buildings, recognize the need to move to improved calculation schemes that are more adequate to real structures.

This need arises due to the fact that the existing methodology for calculating and designing buildings does not solve the problems of the optimal ratio of the sizes of a building box, the diaphragm, floor elements rigidity and so on.

\section{Statement of the Problem}

In this paper, a technique for calculating the continuum plate model of buildings, developed in [7], is proposed. The motion of a building under seismic effect is represented as longitudinal and transverse oscillations of some thick cantilever plate, strained as a three-dimensional body made of relatively soft, low-strength material.

In [7], formulas for the reduced density and moduli of elasticity and shear of a plate model of the building are obtained. Mechanical and physical characteristics of the building are defined under the assumption that the building consists of numerous boxes (rooms) with volumes determined by the formula:

$$
V_{b o x}=d_{1} d_{2} d_{3},
$$

where $d_{1}, d_{2}$-are the dimensions of the building box in plan, $d_{3}$-the height of the box.

Then, to determine the mass of the boxes, the following formula is used

$$
m_{\text {bd }}=\rho_{0} V_{0}=\rho_{\text {bd }} V_{\text {box }},
$$

here $V_{0}$-is the sum of the volumes of bearing and room partitions and floors

$$
V_{0}=2\left(\frac{d_{1} d_{2} h_{3}}{2}+\frac{d_{1} d_{3} h_{2}}{2}+\frac{d_{2} d_{3} h_{1}}{2}\right),
$$

where $h_{1}, h_{2}$-is the thickness of bearing and room partitions. $h_{3}$-is the thickness of the floor.

From relations (1)-(3) a formula for determining the reduced density of a plate model of the building is obtained

$$
\rho_{\mathrm{bd}}=\rho_{0}\left(\frac{h_{3}}{d_{3}}+\frac{h_{2}}{d_{2}}+\frac{h_{1}}{d_{1}}\right) .
$$

Remaining reduced elastic characteristics and densities of the building are determined by the formulas:

$$
\begin{aligned}
& E_{1}^{(\mathrm{bd})}=\xi_{11} E_{0}, E_{2}^{(\mathrm{bd})}=\xi_{22} E_{0}, E_{3}^{(\mathrm{bd})}=\xi_{33} E_{0}, \\
& G_{12}^{(\mathrm{bd})}=\xi_{12} E_{0}, G_{13}^{(\mathrm{bd})}=\xi_{13} E_{0}, G_{23}^{(\mathrm{bd})}=\xi_{23} E_{0}, \rho_{\mathrm{bd}}=\rho_{0} \xi_{0} .
\end{aligned}
$$


where $E_{0}$-is the modulus of elasticity of building material

The values of the coefficients $\xi_{11}, \xi_{22}, \xi_{33}, \xi_{12}, \xi_{13}, \xi_{23}, \zeta_{0}$ for each building cell (room) are defined as functions of two spatial variables, $E_{0}, G_{0}$-are the moduli of elasticity and shear of the strongest bearing panel of the building. Reduced modulus of elasticity of a building is determined by the formula

$$
\begin{gathered}
\xi_{11}=\frac{S_{11}}{S_{01}}, \quad \xi_{22}=\frac{S_{22}}{S_{02}}, \quad \xi_{33}=\frac{S_{33}}{S_{03}} E_{0}, \\
\xi_{12}=\frac{S_{11}}{S_{01}}, \quad \xi_{13}=\frac{h_{\mathrm{fpl}}}{b_{1}} \lambda^{*}, \quad \xi_{23}=\frac{h_{2}}{b}, \\
\zeta_{0}=\frac{V_{1}}{V_{0}} .
\end{gathered}
$$

where $S_{01}, S_{02}, S_{03}$ - are the cross-sectional areas of the building in three coordinate planes of one floor of the building; $S_{11}, S_{22}, S_{33}$-are the total areas of cross sections of the plates in the coordinate planes, forming one floor of the building; $\lambda^{*}$-is a coefficient characterizing the voids in the cross-section of the floor plate, equal to

$$
\lambda^{*}=\frac{a_{1} h_{\mathrm{fpl}}-s^{*}}{a_{1} h_{\mathrm{fpl}}} .
$$

Here $a_{1}$-is a distance between two transverse room partitions plates; $s^{*}$ -is a sum of the voids areas in the cross-section of the floor.

Note that the above volumes and areas are determined, depending on the dimensions of plates, rooms and the building itself, as follows:

$$
\begin{aligned}
& S_{01}=b_{1} H, \quad S_{02}=a H, \quad S_{03}=a b_{1} . \\
& S_{11}=2 b_{1} h_{1}+b_{1} h_{2}+H h_{\mathrm{fpl}}, \\
& S_{22}=2 a h_{1}+a h_{2}+2 H h_{1}+(k-2) H h_{2}, \\
& S_{33}=2 b_{1} h_{1}+a h_{2}+(k-2) b_{1} h_{2} .
\end{aligned}
$$

To represent the values of dynamic characteristics of the plate model of a building, the following formula obtained in [7] is used; it connects the first natural frequency of the plate with mechanical characteristics of material:

$$
E_{\mathrm{bd}}=\left(\frac{\omega_{\mathrm{bd}}}{\omega_{\mathrm{pl}}}\right)^{2} \frac{\rho_{\mathrm{bd}}}{\rho_{\mathrm{pl}}} E_{\mathrm{pl}} .
$$

The values of coefficients $\xi_{11}, \xi_{22}, \xi_{33}, \xi_{12}, \xi_{13}, \xi_{23}$ are determined for each cell (room) of the building. In general, these coefficients are variables and are the functions of two spatial coordinates, which should be determined for the building in question from multiple numerical theoretical experiments and existing experimental data. The moduli of elasticity of continuum plate model of a building are determined by formulas (11).

The equations of longitudinal oscillations of a thick plate [8] [9] [10], built with internal forces and bimoments within the framework of the spatial theory of elasticity, are taken as the equation of motion of a building under seismic action di- 
rected along the longitudinal direction, and are written in the following form

$$
\begin{gathered}
\frac{\partial N_{11}}{\partial x_{1}}+\frac{\partial N_{12}}{\partial x_{2}}=\rho H \ddot{\ddot{\bar{\psi}}_{1}}, \frac{\partial N_{21}}{\partial x_{1}}+\frac{\partial N_{22}}{\partial x_{2}}=\rho H \ddot{\bar{\psi}}_{2}, \\
\frac{\partial T_{11}}{\partial x_{1}}+\frac{\partial T_{12}}{\partial x_{2}}-4 \bar{p}_{13}=\rho H \ddot{\ddot{\beta}_{1}}, \frac{\partial T_{12}}{\partial x_{1}}+\frac{\partial T_{22}}{\partial x_{2}}-4 \bar{p}_{23}=\rho H \ddot{\bar{\beta}}_{2}, \\
\frac{\partial \bar{p}_{13}}{\partial x_{1}}+\frac{\partial \bar{p}_{23}}{\partial x_{2}}-\frac{2 \bar{p}_{33}}{H}=\rho \ddot{\bar{r}}, \frac{\partial \bar{\tau}_{13}}{\partial x_{1}}+\frac{\partial \bar{\tau}_{23}}{\partial x_{2}}-\frac{6 \bar{\tau}_{33}}{H}=\rho \ddot{\bar{\gamma}} .
\end{gathered}
$$

The equations of motion for determining the displacements of external longitudinal walls, obtained by meeting the boundary conditions on the faces of the plate $z=-h$ and $z=+h$ using the method of displacements expansion into the Maclaurin infinite power series, are constructed in [8] [9] [10] in the form:

$$
\begin{gathered}
\bar{W}=\frac{1}{2}(21 \bar{\gamma}-7 \bar{r})-\frac{1}{30} H\left(\frac{E_{31}}{E_{33}} \frac{\partial \bar{u}_{1}}{\partial x_{1}}+\frac{E_{32}}{E_{33}} \frac{\partial \bar{u}_{2}}{\partial x_{2}}\right), \\
\bar{u}_{k}=\frac{1}{4}\left(21 \bar{\beta}_{k}-3 \bar{\psi}_{k}\right)-\frac{1}{20} H \frac{\partial \bar{W}}{\partial x_{k}}+\frac{1}{20} \frac{H \bar{q}_{k}}{G_{k 3}} \quad(k=1,2) .
\end{gathered}
$$

Longitudinal, tangential forces and bimoments are determined with respect to the following nine unknown kinematic functions:

$$
\begin{gathered}
\bar{u}_{k}=\frac{u_{k}^{(+)}+u_{k}^{(-)}}{2}, \quad \bar{\psi}_{k}=\frac{1}{2 h} \int_{-h}^{h} u_{k} \mathrm{~d} z, \quad \bar{\beta}_{k}=\frac{1}{2 h^{3}} \int_{-h}^{h} u_{k} z^{2} \mathrm{~d} z, \quad(k=1,2) \\
\bar{W}=\frac{u_{3}^{(+)}-u_{3}^{(-)}}{2}, \quad \bar{r}=\frac{1}{2 h^{2}} \int_{-h}^{h} u_{3} z \mathrm{~d} z, \quad \bar{\gamma}=\frac{1}{2 h^{4}} \int_{-h}^{h} u_{3} z^{3} \mathrm{~d} z
\end{gathered}
$$

where $u_{i},(i=1,3)$-are the displacements of internal points of the plate; $u_{i}^{(-)}, u_{i}^{(+)},(i=1,3)$-are the displacements of points on the faces of the plate $z=-h$ and $z=+h$. Function $r$ expresses an average value of normal displacements of the plate model of the building; $\bar{W}$-is a half-difference of normal displacements of two outer layers of the plate model; functions $\bar{u}_{1}$ and $\bar{u}_{2}$ -are the half-sums of longitudinal displacements, representing the displacements of the outer layers of the plate model of the building along horizontal and vertical directions.

Expressions of longitudinal and tangential forces are written in the form:

$$
\begin{gathered}
N_{11}=E_{11} H \frac{\partial \bar{\psi}_{1}}{\partial x_{1}}+E_{12} H \frac{\partial \bar{\psi}_{2}}{\partial x_{2}}+2 E_{13} \bar{W}, \quad N_{22}=E_{12} H \frac{\partial \bar{\psi}_{1}}{\partial x_{1}}+E_{22} H \frac{\partial \bar{\psi}_{2}}{\partial x_{2}}+2 E_{23} \bar{W}, \\
N_{12}=N_{21}=G_{12}\left(H \frac{\partial \bar{\psi}_{1}}{\partial x_{2}}+H \frac{\partial \bar{\psi}_{2}}{\partial x_{1}}\right)
\end{gathered}
$$

Expressions of longitudinal and tangential bimoments have the form:

$$
\begin{gathered}
T_{11}=H\left(E_{11} \frac{\partial \bar{\beta}_{1}}{\partial x_{1}}+E_{12} \frac{\partial \bar{\beta}_{2}}{\partial x_{2}}+E_{13} \frac{2 \bar{W}-4 \bar{r}}{H}\right), \\
T_{22}=H\left(E_{12} \frac{\partial \bar{\beta}_{1}}{\partial x_{1}}+E_{22} \frac{\partial \bar{\beta}_{2}}{\partial x_{2}}+E_{23} \frac{2 \bar{W}-4 \bar{r}}{H}\right), T_{12}=T_{21}=H G_{12}\left(\frac{\partial \bar{\beta}_{1}}{\partial x_{2}}+\frac{\partial \bar{\beta}_{2}}{\partial x_{1}}\right) .
\end{gathered}
$$

Bimoments are expressed in the form 


$$
\bar{\sigma}_{11}=E_{11}^{*} \frac{\partial \bar{u}_{1}}{\partial x_{1}}+E_{12}^{*} \frac{\partial \bar{u}_{2}}{\partial x_{2}}, \bar{\sigma}_{12}=G_{12}\left(\frac{\partial \bar{u}_{1}}{\partial x_{2}}+\frac{\partial \bar{u}_{2}}{\partial x_{1}}\right), \bar{\sigma}_{22}=E_{12}^{*} \frac{\partial \bar{u}_{1}}{\partial x_{1}}+E_{22}^{*} \frac{\partial \bar{u}_{2}}{\partial x_{2}} .
$$

where

$$
E_{11}^{*}=E_{11}-E_{31} \frac{E_{13}}{E_{33}}, \quad E_{12}^{*}=E_{12}-E_{32} \frac{E_{13}}{E_{33}}, \quad E_{22}^{*}=E_{22}-E_{32} \frac{E_{23}}{E_{33}}
$$

Intensities of transverse and normal bimoments $\bar{p}_{13}, \bar{p}_{23}, \bar{\tau}_{13}, \bar{\tau}_{23}$ и $\bar{p}_{33}, \bar{\tau}_{33}$ have the form:

$$
\begin{aligned}
& \bar{p}_{k 3}=G_{k 3}\left(\frac{\partial \bar{r}}{\partial x_{k}}+\frac{2\left(\bar{u}_{k}-\bar{\psi}_{k}\right)}{H}\right), \quad \bar{\tau}_{k 3}=G_{k 3}\left(\frac{\partial \bar{\gamma}}{\partial x_{k}}+\frac{2\left(\bar{u}_{k}-3 \bar{\beta}_{k}\right)}{H}\right),(k=1,2) \\
& \bar{p}_{33}=E_{31} \frac{\partial \bar{\psi}_{1}}{\partial x_{1}}+E_{32} \frac{\partial \bar{\psi}_{2}}{\partial x_{2}}+E_{33} \frac{2 \bar{W}}{H}, \bar{\tau}_{33}=E_{31} \frac{\partial \bar{\beta}_{1}}{\partial x_{1}}+E_{32} \frac{\partial \bar{\beta}_{2}}{\partial x_{2}}+E_{33} \frac{2 \bar{W}-4 \bar{r}}{H} .
\end{aligned}
$$

Intensities of bimoments $\bar{\sigma}_{11}^{*}, \bar{\sigma}_{22}^{*}$ are expressed as:

$$
\bar{\sigma}_{11}^{*}=-E_{11} H \frac{\partial^{2} \bar{W}}{\partial x_{1}^{2}}-E_{12} H \frac{\partial^{2} \bar{W}}{\partial x_{2}^{2}}+E_{13} \frac{\bar{R}}{H}, \bar{\sigma}_{22}^{*}=-E_{12} H \frac{\partial^{2} \bar{W}}{\partial x_{1}^{2}}-E_{22} H \frac{\partial^{2} \bar{W}}{\partial x_{2}^{2}}+E_{23} \frac{\bar{R}}{H},
$$

where

$$
\bar{R}=420(\bar{W}+6 \bar{r}-15 \bar{\gamma}) .
$$

The system of differential equations of motion (12)-(16) constitutes a joint system of seven equations with respect to nine unknown functions $\bar{\psi}_{1}, \bar{\psi}_{2}, \bar{\beta}_{1}, \bar{\beta}_{2}, \bar{u}_{1}, \bar{u}_{2}, \bar{r}, \bar{\gamma}, \bar{W}$.

Let us write down the boundary conditions for the problem of bending and shear vibrations of buildings. Suppose that the foundation points move according to a given law $u_{0}(t)$, and the lower part of the building moves horizontally with the foundation. From kinematic considerations it follows that the displacements will be written in the form:

$$
u_{1}\left(x_{1}, 0, z, t\right)=u_{0}(t), u_{2}\left(x_{1}, 0, z, t\right)=u_{3}\left(x_{1}, 0, z, t\right)=0 .
$$

From kinematic conditions it follows that in the foundation of the building the following boundary conditions must be fulfilled:

$$
\bar{\psi}_{1}=u_{0}(t), \bar{\psi}_{2}=0, \bar{\beta}_{1}=\frac{1}{3} u_{0}(t), \bar{\beta}_{2}=0, \bar{r}=0, \bar{\gamma}=0, \bar{u}_{1}=u_{0}(t), \bar{u}_{2}=0, \bar{W}=0 \text {. }
$$

On the free side faces of the building the conditions of zero force factors are fulfilled:

$$
N_{11}=0, N_{12}=0, T_{11}=0, T_{12}=0, \bar{\sigma}_{11}=0, \bar{\sigma}_{12}=0, \bar{p}_{13}=0, \bar{\tau}_{13}=0, \bar{\sigma}_{11}^{*}=0 .
$$

On the free top faces of the building the conditions of zero force factors are fulfilled:

$$
N_{12}=0, N_{22}=0, T_{12}=0, T_{22}=0, \bar{\sigma}_{12}=0, \bar{\sigma}_{22}=0, \bar{p}_{23}=0, \bar{\tau}_{23}=0, \bar{\sigma}_{22}^{*}=0 .
$$

\section{Method of Solution}

The problem is solved by the Finite Differences Method. To approximate the first derivatives, the central difference schemes are taken.

To justify the expressions obtained with the continuous model of calculation, 
let us consider a specific problem with the use of necessary physical and mechanical characteristics of a box-like model. Calculation of the box-like models has been carried out by the FEM, the main point of which is the replacement of a real system (a multi-storey box-like building) with a discrete model of rectangular plane elements (walls and floors) connected at the node points and satisfying the equilibrium conditions of the converging system of forces at each node.

At each nodal point $i$, a part of box mass $m_{i}$ is concentrated, to which convergent forces are applied: the weight $m_{i g}$, the inertia force $P_{i}=m_{i} \ddot{y}_{0}=m_{i} \sin \left(2 \pi \omega_{0} t\right)$ and the elastic force $\left[k_{i j}\right]\left\{q_{j}\right\}$, which is the reaction of this point connection to other points of finite element sampling. Here $\left[k_{i j}\right]$ is the stiffness matrix and $\left\{q_{j}\right\}$ is the displacement vector found as the result of solving the system of differential equations for the entire box

$$
[M]\{\ddot{q}\}+[K]\{q\}=[M]\{g\}+[M]\left\{\ddot{q}_{0}\right\}
$$

The system of differential equations is solved by the Newmark method.

\section{Numerical Results}

Mechanical characteristics and geometry of the rooms panels are accepted as follows: bending bearing panels have a modulus of elasticity $E=20000 \mathrm{MPa}$; density $\rho=2700 \mathrm{~kg} / \mathrm{m}^{3}$; Poisson's ratio $v=0.3$. For the panel working on shear, the following mechanical characteristics are adopted: modulus of elasticity $E=7500 \mathrm{MPa}$, density $\rho=1200 \mathrm{~kg} / \mathrm{m}^{3}$; Poisson's ratio $v=0.3$.

Results of calculations of forced oscillations of a building within the framework of a thick plate model with the following dimensions of the floor slab of the building are: $h_{1}=0.25 \mathrm{~m}, h_{2}=0.25 \mathrm{~m}, h_{\mathrm{fpl}}=0.2 \mathrm{~m}, a_{1}=7.5 \mathrm{~m}, b_{1}=3 \mathrm{~m}$, $a=30 \mathrm{~m}, H=11 \mathrm{~m}$.

In all cases the effect is given by the acceleration of the foundation at amplitude $0.1 \mathrm{~g}$, i.e. at unit amplitude, which corresponds to the intensity of the 7-point earthquake $u_{0}=\sin \left(2 \pi \omega_{0} t\right)$.

The effect frequency is chosen equal to $\omega_{0}=9.5 \mathrm{~Hz}$. Such a high-frequency effect has a period of $T=0.1 \mathrm{~s}$. The Gazli earthquake (1976) accelerogram had such predominant period. So, it can be said that the considered effect is in a certain sense analogous in predominant period to the Gazli earthquake (1976). The scheme of the building in question is shown in Figure 1.

Results obtained with FEM and FDM for the box-like and the continuum plate model of a building are shown in the form of graphs in Figure 2, Figure 3.

Figure 2, Figure 3 show oscillations of characteristic points located at the level of building floors. Solid line characterizes the points at the edge of the floor, and the dashed line-the points at the center of the floor. Under given effects the oscillations of points on the right and left edges of the floor merge.

Figure 2(a) and Figure 2(b) show the displacements of the middle and edge points of the slab on the basis of the plate continuum model of a two-storey building and their values are $\psi_{1}=0.0000374 \mathrm{~m}$ and $\psi_{1}=0.0000449 \mathrm{~m}$, respectively. Figure 2 (c) shows the values of displacements at the middle and edge 


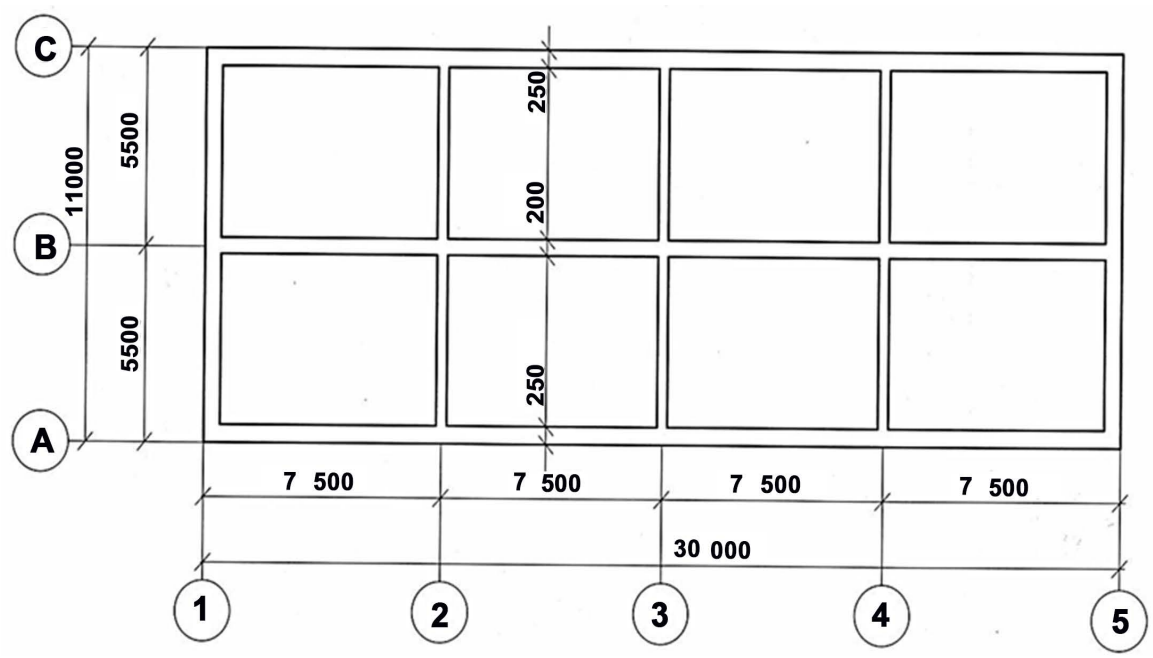

Figure 1. Scheme of the box-like model of a building.

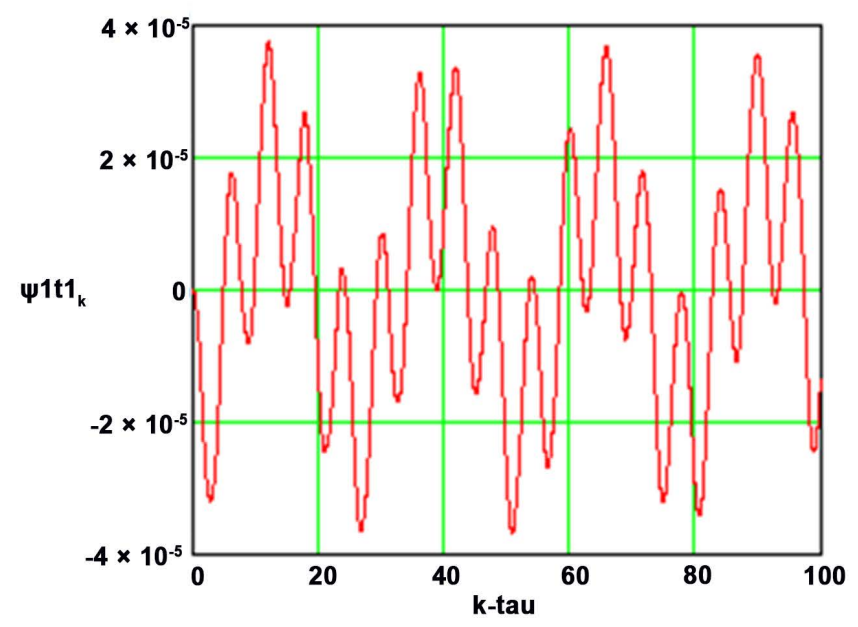

(a)

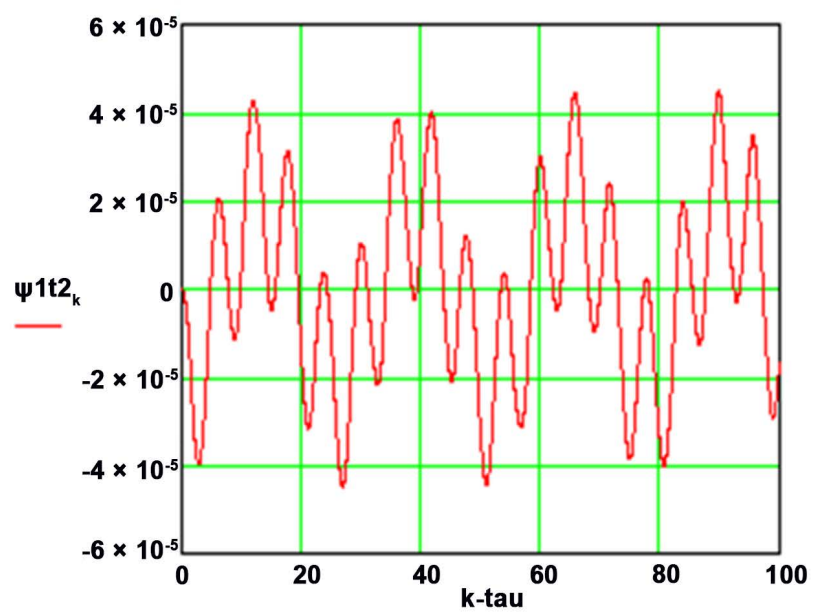

(b)

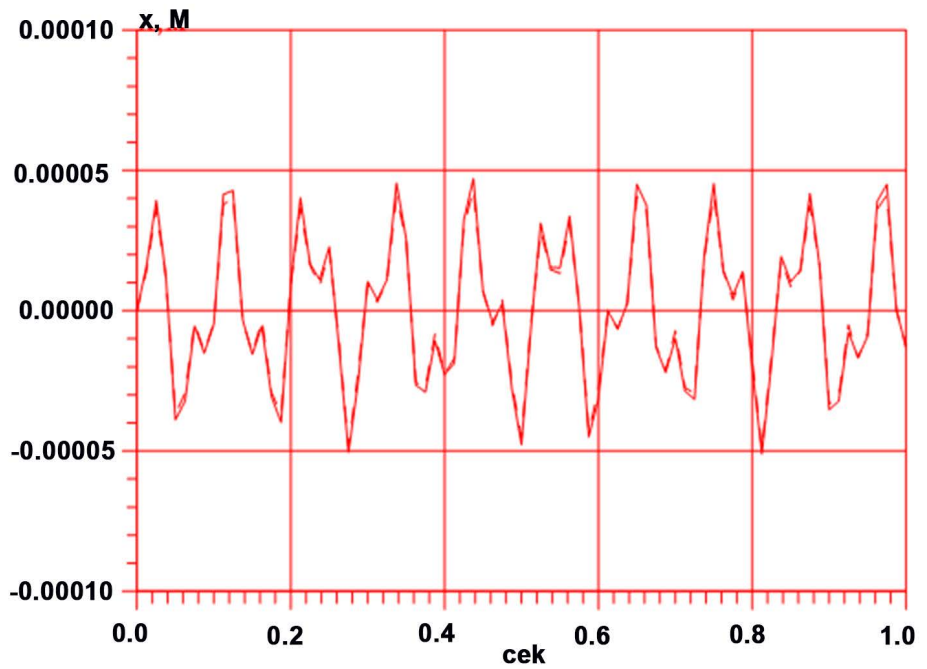

(c)

Figure 2. Displacements of a two-storey building, obtained at characteristic points, in $m$. (a) are the characteristic points at the middle of the floor, (b) are the characteristic points at the edge of the floor, (c) is the graph obtained with FEM. 


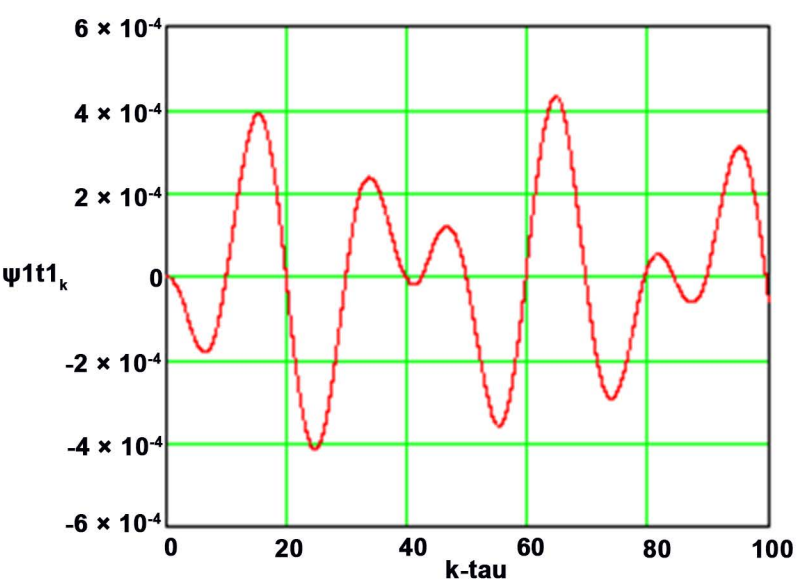

(a)

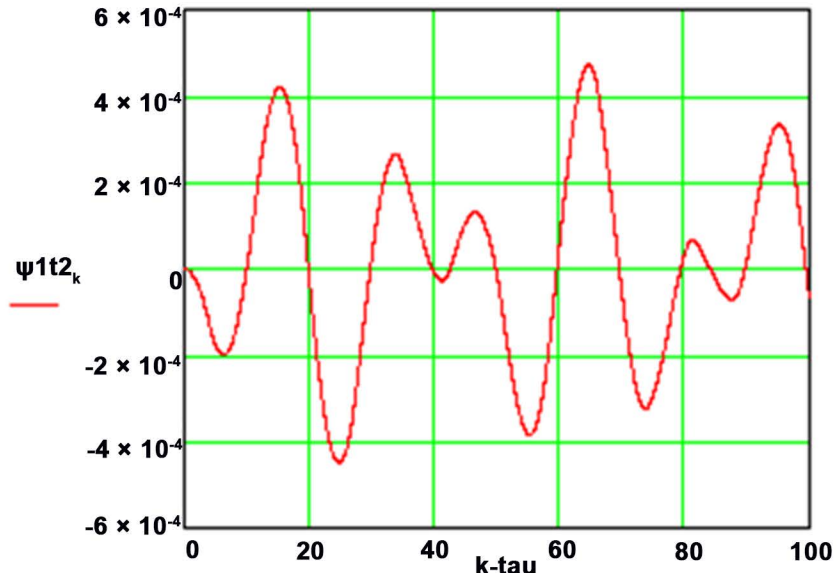

(b)

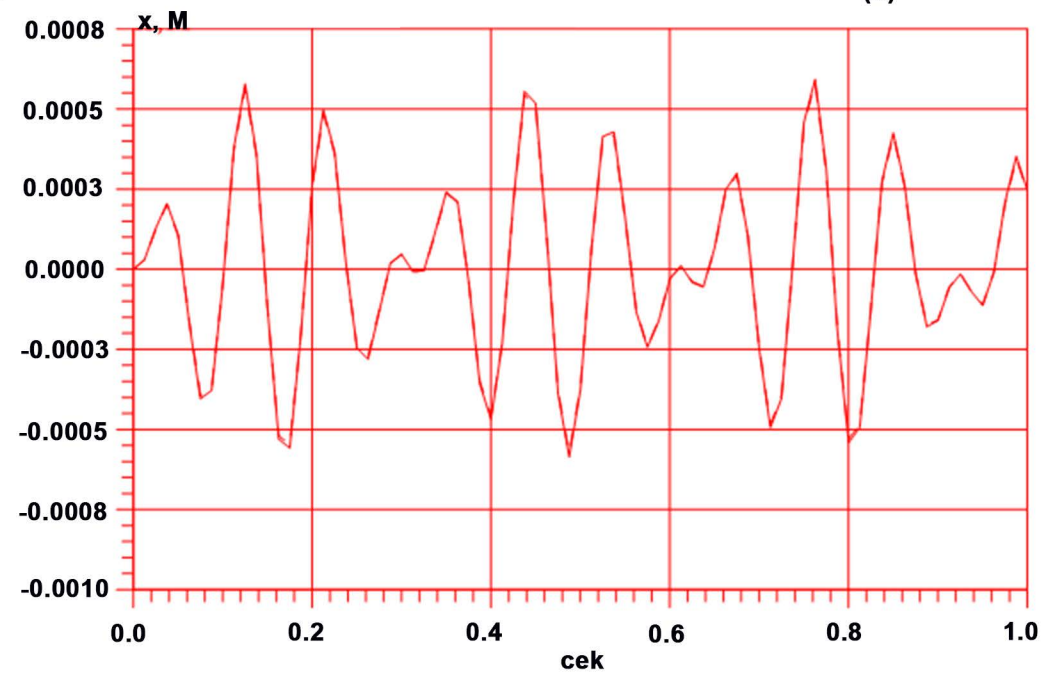

(c)

Figure 3. Displacements of a five-storey building, obtained at characteristic points, in $m$ (a) is the characteristic point at the middle of the floor, (b) is the characteristic point at the left edge of the floor, (c) is the graph obtained with FEM.

points of the slab on the basis of the box-like model with FEM of a two-storey building and their values are $R_{\text {mid }}=0.0000430 \mathrm{~m}$ and $R_{\text {edg }}=0.0000500 \mathrm{~m}$, respectively.

Comparison of results based on the plotted graphs shows that the values of displacements based on the box-like model with FEM are 10\% - 13\% less than the results based on the plate continuum model.

Figure 3(a) and Figure 3(b) show the displacements at the middle and edge points of the slab on the basis of the plate continuum model of a five-story building and their values are $\psi_{1}=0.000472 \mathrm{~m}$ and $\psi_{1}=0.000496 \mathrm{~m}$, respectively. Figure 3(c) shows the graph of displacements at the middle and edge points of the slab on the basis of the box-like model with FEM of a five-storey building and their values are $R_{\text {mid }}=0.000520 \mathrm{~m}$ and $R_{\text {edg }}=0.000550 \mathrm{~m}$.

When comparing results based on plotted graphs for the box-like model with FEM and the plate continuum model, it can be seen that the values of displace- 
ments do not differ significantly.

The values of the generalized displacements obtained from the calculations of two-storey and five-story buildings for seismic effects at different points of floor differ within the range of $10 \%$.

\section{Conclusion}

In conclusion, it should be noted that on the basis of the bimoment theory of thick plates a dynamic plate-like model of the building has been developed, reflecting its spatial strains. Using the geometry of the building and its rooms, the reduced density, moduli of elasticity and shear of the plate model have been determined. The plate model of the building due to the choice of coefficients $\xi_{22}, \xi_{33}, \xi_{12}, \xi_{13}, \xi_{23}$, adequately reflects the laws of displacements of the points of the building. So, the plate model is acceptable for determining the displacement fields of a tall building during an earthquake and the dynamic characteristics of a building.

\section{Conflicts of Interest}

The authors declare no conflicts of interest regarding the publication of this paper.

\section{References}

[1] Rashidov, T.R., Shamsiev, U.S., Musheev, R.N. and Bovshover, A.Z. (1992) Seismodynamics of Spatial Systems. "Fan", Tashkent, 152 p.

[2] Abdurashidov, K.S. (1989) Oscillation and Seismic Stability of Industrial Structures. "Fan", Tashkent, $76 \mathrm{p}$.

[3] Birbraer, A.N. (1998) Calculation of Structures for Seismic Resistance. Nauka, St. Petersburg, $254 \mathrm{p}$.

[4] Ramin, K. and Mehrabpour, F. (2014) Study of Short Column Behavior Originating from the Level Difference on Sloping Lots during Earthquake (Special Case: Reinforced Concrete Buildings). Open Journal of Civil Engineering, USA, 4, 23-34. https://doi.org/10.4236/ojce.2014.41003

[5] Corchete, V. (2010) The Analysis of Accelerograms for the Earthquake Resistant Design of Structures. International Journal of Geosciences, 32-37. https://doi.org/10.4236/ijg.2010.11004

[6] Al-Ansari, M.S. (2013) Reliability Index of Tall Buildings in Earthquake Zones. Open Journal of Earthquake Research, 2, 39-46. https://doi.org/10.4236/ojer.2013.23005

[7] Usarov, M.K., Ayubov, G.T. and Usarov, D.M. (2017) Dynamic Calculation of the Building on the Basis of a Plate Model, Taking into Account the Bimoments. Problems of Mechanics, No. 1, 34-39.

[8] Usarov, M.K. (2015) Bending of Orthotropic Plates with Consideration of Bimoments. Engineering and Construction Magazine, №1, 80-90.

[9] Usarov, M.K. (2014) Bimoment Theory of Bending and Oscillation of Thick Orthotropic Plates. Bulletin of the NUUz, No. 2/1, 127-132.

[10] Usarov, M.K. (2016) Dynamic Design of Thick Orthotropic Cantilever Plates with Consideration of Bimoments. World Journal of Mechanics, 6, 341-356.

https://doi.org/10.4236/wjm.2016.610025 